\title{
Effects of High-Fat Diet and Treadmill Running on The Hypothalamic Kiss-1-GPR54 Signaling Pathway in Growing Male Rats
}

RUI XU

Nanjing Sport Institute https://orcid.org/0000-0001-6120-0928

Junpeng FENG

Beijing Sport University

Chunyu LIANG

Beijing Sport University

Ge SONG

Beijing Sport University

Yi YAN ( $D$ yanyi22@sina.com )

Beijing Sport University

\section{Research}

Keywords: kiss-1-GPR54, high-fat diet, exercise, growing period, HPT axis

Posted Date: July 27th, 2021

DOI: https://doi.org/10.21203/rs.3.rs-717653/v1

License: (c) (1) This work is licensed under a Creative Commons Attribution 4.0 International License. Read Full License 


\section{Abstract}

Background: Kiss-1 neurons, one of the metabolic sensors in the hypothalamic, is necessary for puberty initiating and acts through G protein-coupled receptor, known as GPR54. This study investigated the mechanism of the hypothalamic Kiss-1-GPR54 signaling pathway in high-fat diet and exercise on the growing male rats.

Methods: 135 three-week-old weaned male rats were underwent high-fat diet and exercise (60-70\% $\mathrm{VO}_{2 \mathrm{max}}, 1 \mathrm{~h} /$ day, 5 days/ week). These mice were randomly divided into control group, control and exercise group, high-fat diet group, high-fat diet and exercise group. Hypothalamic, testis, and serum samples of each group were collected at PND (postanal day)21st day (21D, early childhood), PND 43rd day (43D, puberty) and PND 56th day (56D, maturity). Immunofluorescence, Quantitative real-time PCR, hematoxylin and eosin staining, chemiluminescent immunoassays were used in studies. Analysis of variance (ANOVA) was used to analyze the effects of age (PND 21,43,56), exercise (exercise, sedentariness), and diet (high-fat, normal) on the biological indexes in rats

Results: The mRNA and protein expression of Kiss-1, GPR54 in the hypothalamic were gradually increased along with growing and peaked at PND 43, and the serum testosterone increased and peaked at PND 56. High-fat diet increased the expression of Kiss-GPR54 system in hypothalamic, while the serum testosterone decreased during different stages of growth. Exercise decreased the expression of Kiss-1 at PND 56 and increased the expression of Kiss-1at PND 43, meanwhile decreased testosterone and the deposition of lipid droplets in the testis at all age of development.

Conclusions: The expression of Kiss-1-GPR54 in male rats showed fluctuated change during growth and development. High-fat diet can upregulate the expression of Kiss-1-GPR54 system in hypothalamic. Exercise can correct the adverse effect of high-fat diet on Kiss-1-GPR54 signaling pathway in hypothalamic and the function of hypothalamic-pituitary-testicular (HPT) axis, upregulate Kiss-1 at puberty and downregulate of Kiss-1 at maturity of high-fat diet male rats.

\section{Introduction}

Puberty is an important developmental stage which requires maturation of the reproductive neuroendocrine axis and subsequent initiation of high-frequency, episodic release of gonadotropinreleasing hormone (GnRH) [1].Overfeeding or high-fat diet can induce precocious puberty in both boys and girls [2], which correlates with body weight and body mass index (BMI). Childhood obesity disrupts pubertal onset of children and lower the future life quality of children further more.

The Kiss-1 gene and its receptor gene GPR54 in the hypothalamic, especially in the arcuate nucleus (ARC) play a key role in the initiation of puberty [3] Kiss-1-GPR54 system can activate the phospholipase-C- $\beta$ (PLC $\beta$ ) pathway $[4,5]$, and then cause depolarization of hypothalamic GnRH neurons, and then stimulate luteinizing hormone $(\mathrm{LH})$ and follicle-stimulating hormone (FSH) secretion. It is reported that the 
pubertal rats [6, 7], whereas the expression of hypothalamic Kiss-1 and the secretion of LH and FSH from the hypothalamic-connected pituitary gland obviously decreased in fasting female rats[8]. The negative energy balance due to subnutrition could induce puberty delay through suppressing ARC Kiss-1neuron [9].

Exercise has attracted broad attention for reducing energy accumulation and decreasing body fat production. Recent studies reported that exercise could be an effective measure to prevent childhood obesity [10] and central precocious puberty[11]. The Kiss-1/GPR54 system plays an important role in puberty-initiated control. However, whether exercise can alter the high-fat diet induced precocious puberty through Kiss-1-GPR54 signaling pathway in hypothalamic has yet to be determined.

In order to answer these questions, this study examined the effects of high-fat diet and moderateintensity treadmill training on the hypothalamic Kiss-1-GPR54 signaling pathway and their following effects on reproductive organs in the growing male rats.

\section{Materials And Methods}

\subsection{Animals}

Animal experiments were approved by the Animal Welfare Ethics Committee of Beijing Sport University (approval number: 2016021A). 135 weaned male Sprague-Dawley rats at PND $21(58.5 \pm 2.7 \mathrm{~g})$ were purchased from Beijing Vital River Laboratory Animal Technology Company Limited (license number: SCXK 2012-0001; Beijing, China). Rats were kept under a controlled room temperature $\left(25 \pm 0.5^{\circ} \mathrm{C}\right)$ with a 12-h light/12-h dark cycle. Five rats were housed per cage and ad libitum accessed food and water.

\subsection{Experimental design}

Animals were randomly divided into four groups: a control group $(C, n=45)$, a normal diet with exercise group ( $C E, n=30)$, a high-fat-diet control group $(H C, n=30)$, and high-fat-diet with exercise group $(H E, n=$ 30 ). The $C$ and $C E$ group were fed with a normal diet (20\% protein, $70 \%$ carbohydrate, $10 \%$ fat, total heat was $3.85 \mathrm{kcal} / \mathrm{g}$; Product ID: D12450B). The HC and HE groups were fed with a high-fat diet (20\% protein, $35 \%$ carbohydrate, $45 \%$ fat, total heat was $4.73 \mathrm{kcal} / \mathrm{g}$; Product ID: D12451). All food products were purchased from Beijing HFK Bioscience, China.

\section{Exercise training}

Rats in CE and HE group were trained with an intensity of $60-70 \%$ of the maximum oxygen uptake $\left(\cdot \mathrm{VO}_{2 \max }\right)$ for $60 \mathrm{~min} /$ day, 5 days per week (rat treadmill, DSPT-208; Hangzhou Segment Manufacturing, China).We chose this range because $60-70 \%$ the $\cdot \mathrm{VO}_{2 \max }$ represents moderate-intensity exercise, which has been shown to be the best exercise intensity for fat consumption [12]. In the first three days adaptive training (speed $10 \mathrm{~m} / \mathrm{min}$, 0-degree incline), the durations were $10 \mathrm{~min}, 20 \mathrm{~min}$, and $30 \mathrm{~min}$. After the adaption period, the incline was raised to 10-degrees for the duration of the training period. The treadmill traininn nrannoc wanc dividnd inta thn fallowaina thron ctanes: (1) a warm-up period (5 min), (2) training Loading [MathJax]/jax/output/CommonHTML/fonts/TeX/fontdata.js 
period (50 $\mathrm{min}$ ), and (3) cool-down period (5 min). During the warm-up period and the cool-down period, the running speed was set at $10 \mathrm{~m} / \mathrm{min}$, while the running speed during the training period was $60-70 \%$ $\cdot \mathrm{VO}_{2 \max }(\mathrm{V}=12 \sim 17 \mathrm{~m} / \mathrm{min})$. Treadmill speed of training period was gradually increased with the capacity improves, which were determined according to the corresponding speeds of $100 \% \cdot \mathrm{VO}_{2 \max }$ of rats at different ages.

\section{Tissue collection}

Rats were sacrificed at 21st postnatal day $(n=15)$, 43rd postnatal day $(n=60)$, and 56th postnatal day ( $n$ $=60$ ), there were include C, CE, HC and HE group at PND 43 and PND 56. 3 rats in each group received a thoracotomy and were perfused through the left ventricle with of saline followed by $4 \%$ paraformaldehyde after deep anesthetization. The brains were removed and immersed into $4 \%$ paraformaldehyde for a $24 \mathrm{~h}$ post-fixation period then placed in $75 \%$ ethanol, dehydrated in an ascending series of gradient ethanol cleared in xylene, and embedded in paraffin for subsequent immunofluorescence.

Another 12 rats rapidly anesthetized with $2 \%$ sodium pentobarbital $(0.25 \mathrm{ml} / 100 \mathrm{~g}$ body weight), abdominal aortic blood was centrifuged to collect serum for serum hormonal test, hypothalamic and one side of testis were frozen immediately in liquid nitrogen and stored at $-80^{\circ} \mathrm{C}$ for quantitative real-time PCR (qRT-PCR) after euthanized, contralateral testis were immediately removed and immersed into $4 \%$ paraformaldehyde for a $24 \mathrm{~h}$ post-fixation period then dehydrated in an ascending series of gradient ethanol, and embedded in paraffin for subsequent hematoxylin and eosin (HE) staining.

\subsection{HE staining}

Testis tissues were excised and fixed overnight in 4\% paraformaldehyde and embedded in paraffin. Sections (4- $\mu \mathrm{m}$ thick) were cut and subjected to hematoxylin and eosin (HE) staining for histological analysis. Digital images (200x) were captured from five random fields per section using a Leica microscope.

\subsection{Hormonal Assays}

The testosterone contents in serum samples were measured via chemiluminescent immunoassays (CLIAs) using a Beckman Coulter UniCel DxI 800 Access Immunoassay System (Beckman Coulter, USA).

\section{5 qRT-PCR Analysis}

Total RNA was extracted from the hypothalamic using an Animal Tissue RNA Extraction Kit (Tiangen, China) according to the manufacturer's protocol. RNA was reverse transcribed using the Toyobo ReverTra Ace qPCR RT kit (Toyobo, Japan). Then, cDNA was stored at $-20^{\circ} \mathrm{C}$ until further use. Subsequently, qRTPCR was performed using a Bio-Rad iCycler Detection System and a Quantitect SYBR Green PCR kit (TaKaRa, Japan). Primers sequences were as follow: Kiss-1 (F: ATGATCTCGCTGGCTTCTTG, R: CTGTGGGTTCAGGGTTCAC); GPR54 (F: CCACATGTGCCACTTTGACA, R: AACCCACCCAGATGCTAAGG); 
(F:CCGCTGTTGTTCTGTTGACT, R: GCAGATCCCTAAGAGGTGAA). Standard curves were generated, and $\beta$ actin was used as a housekeeping gene to quantify the abundance of CDNA in each sample. The qRTPCR cycling parameters were as follows: $95^{\circ} \mathrm{C}$ for $15 \mathrm{~min}$; and 40 cycles of $94{ }^{\circ} \mathrm{C}$ for $15 \mathrm{sec}, 60{ }^{\circ} \mathrm{C}$ for $30 \mathrm{sec} 72{ }^{\circ} \mathrm{C}$ for $30 \mathrm{sec}$. Data collection was chosen at the $72{ }^{\circ} \mathrm{C}$ extension phase. To ensure the presence of a single product, a dissociation curve was performed after each run. Data were collected from threshold values using the automatic function of the Bio-Rad MylQ software. All samples were run in duplicate. The data for Kiss-1, GPR54, PLC, GnRH mRNA were normalized to that of $\beta$-actin.

\subsection{Immunofluorescence double labeling and confocal microscopy}

Hypothalamic tissue blocks were dehydrated in an ascending series of ethanol, cleared in xylene, and embedded in paraffin. Immunofluorescent staining was used to observe the expression of Kiss-1 and GPR54 proteins in the ARC of hypothalamic of each group. Hypothalamic sections (10- $\mu \mathrm{m}$ in thickness) were deparaffinized, and antigen retrieval was performed with $0.01 \mathrm{M}$ of sodium citrate buffer. An endogenous peroxidase blockade was performed with $3 \%$ hydrogen peroxidase, and nonspecific binding was inhibited. Sections were incubated overnight in Kiss-1 antibody (1:500, Millipore, \# NP-002247) primary antibody, and then incubated with Alexa Flour 594 conjugate $(1: 800$, Cell Signaling, USA \# Antirabbit lgG $[H+L]$, red) for $1 \mathrm{~h}$ at room temperature. Then incubated overnight in GPR54(1:200, Novus, USA \# NLS1926) primary antibody in the same sections, and incubated with Alexa Flour 488- conjugated streptavidin (1:300, Jackson ImmunoResearch, USA \# 131489, green) for $1 \mathrm{~h}$ at room temperature. Finally, the sections were counterstained with DAPI to pinpoint the cell nucleus.

Confocal laser microscopy was performed with excitation wavelengths of 488 and $594 \mathrm{~nm}$, from which protein fluorescence was visualized. We analyzed at ARC (interaural from $7.2 \mathrm{~mm}$ to $5.64 \mathrm{~mm}$, bregma from $-1.8 \mathrm{~mm}$ to $-3.36 \mathrm{~mm}$ )[13], location by using the third ventricle. Each section was collected according to the same parameters, and the position of the ARC was determined in a 200 -fold field first and a 400fold field was then used to select six fields of view imaging and quantification of the number of positively stained cells in each field of view.

\subsection{Statistical Analysis}

All data are presented as mean \pm standard deviation (SD). Analysis of variance (ANOVA) was used to analyze the effects of age (PND 21,43,56), exercise (exercise, sedentariness), and diet (high-fat, normal) on the biological indexes in rats. Three-factor variance analysis was used to determine whether there was a three-factor interaction among independent variables, in which case a simple two-factor analysis was continued. Alternatively, if there was a two-factor interaction, a simple independent-effect test was used. Statistical analysis was carried out with SPSS version 21.0 (SPSS Inc., Chicago, IL, USA). $P<0.05$ was considered to be statistically significant.

\section{Results}




\section{Changes of Kiss-1-GPR54 signaling pathway of growing rats}

To determine whether Kiss-1-GPR54 signaling pathway affected by age $(P<0.01)$, we measured Kiss-1, GPR54, PLC mRNA levels in the hypothalamic. We found similar variation tendency in Kiss-1, GPR54, and PLC mRNA in the hypothalamic during development, it was fluctuated with the growth and development in male rats. All of the three genes first increased and then decreased from PND 21 to PND 56, and reached a peak at PND 43. Additionally, we analyzed Kiss-1 protein expression in the rat hypothalamic ARC via immunofluorescence, we found that the protein expression of Kiss-1 also reached a peak at PND 43, which was consistent with mRNA. But the change of GPR54 protein did not change during development was contrast with Kiss-1 protein. The number of GPR54 protein was more than Kiss-1 protein throughout the developmental process (Fig. 3).

\section{Effect of high-fat-diet on the expression of Kiss-1-GPR54 signaling pathway of growing rats}

As shown in Fig. 2B, we found that high-fat diet intervention upregulated the mRNA expression of Kiss-1 in the hypothalamic. Compared with $\mathrm{C}$ group, it was shown a significantly increased at PND 56 after highfat diet intervention (Fig. 2B, $\mathrm{P}<0.05$ ). Furthermore, a high-fat diet increased the protein expression of Kiss-1 in the ARC than in the $C$ group at both PND 43 and PND 56 (Fig. 4B, $P<0.05$ ).

PLC is downstream of the Kiss-1 system, as a key factor in stimulating voltage-gated calcium channels. As shown in Fig. 2E and 2F, the expression of PLC mRNA at PND 43 was significantly higher than other period, high-fat diet decreased the expression of PLC mRNA in hypothalamic, especially at PND 43.

\section{Effect of exercise on the expression of Kiss-1-GPR54 signaling pathway of growing rats}

To investigate whether the effect of exercise intervention on high-fat diet male rats of different ages are consistent, we examined expression of Kiss-1-GPR54 signaling pathway at the age of PND 43 and PND 56 in both CE and HE groups. We found that the expression of Kiss-1 mRNA in the hypothalamic at PND 43 was significantly increased after continuous $60-70 \% \cdot \mathrm{VO}_{2 \max }$ treadmill training in high-fat diet rats (Fig. 2B, P < 0.05). The mRNA levels in GPR54 in the hypothalamic were significantly increased in both CE and $\mathrm{HE}$ groups after exercise intervention (Fig. 2D, $\mathrm{P}<0.05$ ). Exercise intervention could upregulate Kiss-1GPR54 signaling pathway in high-fat diet rats at PND 43. But for maturity rats, exercise intervention showed a different result. As shown in Fig. 2B, we found that exercise intervention downregulated the expression of Kiss-1 mRNA and protein in high-fat diet rats at PND 56, which was the maturity period (Fig. 2B, 2H, 2I, P<0.05,). The expression of GPR54 protein in the ARC of the hypothalamic in the HE group was also remarkable lower than that in the HC group at PND $56(P<0.05$, Fig. 4D, 4E, 4I).

\section{Effect of high-fat diet and exercise on body mass and reproductive system of male rats}

Body weight and body length increased gradually with growth in all groups. High-fat diet intervention significantly upregulated body mass and body length at PDN $43(P<0.05$, Fig. 5A, 5B), but not at PND 56 $(P>0.05)$. Exercise intervention significantly downregulated body mass and body length at PND 56 in 
standard diet rats $(P<0.05)$, and significantly reduced body mass at PND 56 in high-fat diet rats $(P<$ $0.05)$. We also found that the body mass and body length showed a tendency to increase after exercise intervention in standard diet rats at PDN 43, which was consistent with then change of Kiss-1.

In $\mathrm{C}$ group, we found the testicular mass was significantly increased with the rise of body mass and body length in all groups. The results of HE staining also showed that with the development of the testes, the number of sperm cells and spermatocytes increased in $C$ group of different age stage (Fig. 6A, 6B, 6C), meanwhile, the serum testosterone levels increased at all stages of development from childhood to maturity as the morphology of testis matured, which were significantly increased at PND 56 (Fig. 5D, P< 0.05).

After high-fat diet or by $60-70 \% \cdot \mathrm{VO}_{2 \max }$ treadmill training intervention, the weight of the testes did not show any change (Fig. 5C), but HE staining in high-fat diet rats showed that the testicular lipid droplets increased, while, the number of spermatozoa and leydig cells in the testicular decreased. From the staining results, we found that the epithelium of spermatogenic cells were arranged loosely, and in the seminiferous tubules, vacuoles formed between spermatocytes and spermatoblasts due to the deposition of lipid droplets (Fig. 6F, 6G). The serum testosterone levels decreased in high-fat diet rats $(P<0.05$, Fig. 5D), but it was mainly occurred at PND 43. Exercise intervention could increase serum testosterone levels in high-fat diet rats (Fig. 5D, $\mathrm{P}<0.05$ ), but it also reduced serum testosterone levels in standard diet rats, especially at PND 56. Meanwhile the results of HE staining showed that the vacuoles formed between spermatocytes and spermatoblasts decreased with exercise, and the number of leydig cells in the HE group was also increased compared with those in the HC group (Fig. $6 \mathrm{H}, 6 \mathrm{l}$ ).

\section{Discussion}

In this study, we measured the growth and development of male rats, as well as parameters of the hypothalamic Kiss-1-GPR54 signaling pathway, at three time points: (a) PND 21 st day (21D, early childhood); (b) PND 43rd day (43D, puberty), and (c) PND 56th day (56 D, maturity). Normally the body mass, body length and reproductive organs (testis) weight, serum testosterone of rats gradually increased during development. The expression levels of Kiss-1-GPR54 signaling pathway exhibited non-linear dynamics across their growing. Kiss-1, GPR54 and their downstream factors PLC increased continuously from PND 21 and reached a peak at PND 43, and they had decreased measurably at PND 56. In our previous studies on high-fat diet and exercise on testis of growing male rats, the GSI which symbolizes the reproductive development of rats also showed this trend[14], we suggested that the expression of Kiss- 1 in hypothalamic was closely related to reproductive function. Takumi et al. measured the relative expression of Kiss- 1 in the hypothalamic of rats at 3, 4, 5, 6, and 8 weeks old, and found that Kiss-1 expression increased gradually from three to five weeks old, and then decreased obviously at eight weeks old, especially in female rats [15]. The change of Kiss-1 is consistent with the results of our present study first increase and then decrease across development. So it is possible that Kiss-1 in hypothalamic was fluctuated with growing both in male and female rats. The reason for this discrepancy may be that the 
specific time of pubertal onset and the peak time of Kiss-1 expression are slightly different between males and females, as this previous study used female rats.

GPR54 as a receptor of Kiss-1, it was a membrane-bound protein expressed on GnRH neurons, Kiss-1 regulates GnRH through GPR54. In this study, we found that although the expression of GPR54 mRNA was consistent with that of Kiss-1 during development, but there were large amounts in ARC by immunofluorescence technique. We suggest that a series of reactions contributing to progression of GPR54 gene transcription into protein during growth and development and Kiss-1 play a key regulatory role in the Kiss-1-GPR54 signaling pathway.

Increasing evidence shows that the onset of puberty is largely dependent on body weight rather than chronological age [9]. In order to investigate the effects of a high-fat diet on Kiss-1-related indexes and metabolism in the hypothalamic of adolescent rats, our present study established a high-fat diet model rather than an obesity model, rats were given a high-fat diet with a 45\% fat content starting at PND 21 . Our approach yielded an energy-imbalance model in which a high-fat diet could induce positive energy balance in male rats. Energy status was commonly linked to altered onset of puberty and reproductive impairment [16]. It has been reported that high fat diet could induce female rats show obesity and precocious puberty (Takumi et al., 2015), and induce earlier puberty in obese girls[17].

In this study, we showed that high-fat diet intervention significantly increased the expression of Kiss-1 in the ARC in male rats at PND 43 and PND 56, it means that high-fat diet was also effected on the Kiss-1 system in hypothalamic in male. Moreover, we demonstrate that the effect of high-fat diet not only on puberty stage, but also on maturity stage. Meanwhile, Chantacha et al. also found that obese men serum kisspeptin was positively correlated with body weight, serum kisspeptin levels were significantly in obese men[18].It means the expression of Kiss-1 in hypothalamic in male at maturity could affected by positive energy balance, such as high-fat diet and obesity. Another interesting finding of this study is that although no significant increase in body mass in a short-term high-fat diet intervention from PND 21 to PND 43, the expression of Kiss-1 in hypothalamic also increased. The work of Ullah et al in male mouse supports our present finding that high-fat-diet could induced precocious puberty even in normal body weight mouse[1]. Furthermore, we show that although the expression of Kiss-1 increased after high-fat diet intervention, it still maintained the change trend during the development process of male rats, which increased first and then decreased, and peak at 43D, and the GSI curve also do not change during development[14]. We demonstrate that testes weight and GSI did not show any change after high-fat diet, but serum testosterone levels and testicular morphology was affected. The function of reproductive organs is regulated by HPG axis, especially leydig cells, the number of leydig cells decreased is accompanied by a decrease in serum testosterone levels. Another study of our team also found that the Kiss-1 system in testes tissue was also affected by high-fat diet, and it significantly correlated with testicular tissue testosterone levels, all these findings suggest the reproductive organs is regulated by Kiss-1 in the hypothalamic or testes, and the correlation is to be further explored. 
Energy imbalance can induce the body to undergo a series of stress and/or adaptative responses. As a form of energy consumption, our present study revealed the effect of $60-70 \% \cdot \mathrm{VO}_{2 \text { max }}$ treadmill training as an intervention on male rats at different developmental stages. We demonstrate that the expression of Kiss-1 and GPR54 in HE group was decreased accompanied with body mass and body length decreased after moderate-intensity exercise, meanwhile the number of leydig cells in testes and serum testosterone levels also improved, the GPR54 protein in testes of high-fat diet rats also increased after exercise intervention. Similarly, it has been reported that $72 \mathrm{~h}$ deprivation could induced the expression of Kiss-1 mRNA were significantly lower in adult female rats [19]. Most of these changes were found to occur at PND 56, suggest that negative energy balance could decreased the expression of Kiss-1 in hypothalamic in both male and female at maturity stage.

It is interesting to note that moderate-intensity exercise induced a different result at puberty period, we demonstrate that the expression of Kiss-1 in hypothalamic was increased after moderate-intensity exercise at PND 43 which was the usual time of puberty in male rats. Previously it was reported that calorie restriction modulates delayed reproductive development in pre-pubertal male rats[20]. However, the changes caused by fasting are not the same as those caused by exercise. This distinction may be because exercise is not only a means for regulating energy consumption, but is also a means for regulating metabolism, which can stimulate the secretion of hormones, such as growth hormones and sex hormones, although the weight of rats decreased, their body lengths increased after exercise intervention. Increasing evidences showed that puberty onset is largely dependent on body mass rather than chronological age[21, 22]. We suggested that moderate exercise may stimulate growth and development in puberty rats and the increased the expression of Kiss-1/GPR54 system. This feature is distinct from that of other regulators of negative energy balance, such as fasting, nutritional restriction, and cold environments. After our exercise intervention we found that serum testosterone levels increased, and lipid droplets in the testes decreased in the HE group at PND 43, which is the puberty period in male rats. It has been reported that $\mathrm{T}$ concentration were lower in obese early puberty boys, and aerobic exercise could increase the T concentration[23]. All these findings show that exercise intervention can correct the adverse effects of high-fat diet on hypothalamic and testes in male rats.

\section{Conclusion}

1) Kiss-1-GPR54 signaling pathway in the hypothalamic of male rats were fluctuated during development. It was increased first, peaked at PND 43, and then decreased.

2) High-fat diet can inhibit the reproduction development, upregulate the expression of Kiss-1 in hypothalamic in the growth phase of male rats.

3) $60-70 \% \cdot \mathrm{VO}_{2 \max }$ treadmill training can correct the adverse effect of high-fat diet on the function of testicular, upregulate the expression of Kiss-1 in hypothalamic at puberty period, and downregulate the expression of Kiss-1 in hypothalamic at maturity period. 


\section{Abbreviations}

PND: Postanal day; HPT: Hypothalamic-pituitary-testicular; GnRH: Gonadotropin-releasing hormone; BMl: Body mass index; $\mathrm{V}_{2 \max }$ : Maximum oxygen uptakeGPR54: $\mathrm{G}$ protein-coupled receptor; ARC: Arcuate nucleus; PLC $\beta$ : Phospholipase-C- $\beta$; GnRH: Gonadotropin-releasing hormone; LH: Luteinizing hormone; FSH: Follicle-stimulating hormone.

\section{Declarations}

\section{Ethics approval and consent to participate}

Animal experiments were approved by the Animal Welfare Ethics Committee of Beijing Sport University (approval number: 2016021A).

\section{Consent for publication}

Not applicable.

\section{Availability of data and materials}

The datasets used and/or analysed during the current study are available from the corresponding author on reasonable request.

\section{Competing interests}

The authors declare that they have no competing interests.

\section{Funding}

We are thankful for the support provided by the National Natural Science Foundation of China (No. 32071173), the Natural science fund for colleges and universities in Jiangsu Province (19KJB180018).

\section{Authors' contributions}

Conceived and designed the experiments: XU Rui, YAN Yi, FENG Junpeng. Performed the experiments: XU Rui, FENG Junpeng, LIANG Chunyu, SONG Ge. Analyzed the data: XU Rui, YAN Yi. All authors read and approved the final manuscript.

\section{Acknowledgements}

We are thankful for the support provided by the National Natural Science Foundation of China (No. 32071173), the Natural science fund for colleges and universities in Jiangsu Province (19KJB180018). 
1. Ullah R, Su Y, Shen Y, Li C, Xu X, Zhang J, Huang K, Rauf N, He Y, Cheng J, et al. Postnatal feeding with high-fat diet induces obesity and precocious puberty in C57BL/6J mouse pups: a novel model of obesity and puberty. Frontiers of medicine. 2017;11(2):266-76.

2. Ullah R, Raza A, Rauf N, Shen Y, Zhou YD, Fu J. Postnatal Feeding With a Fat Rich Diet Induces Precocious Puberty Independent of Body Weight, Body Fat, and Leptin Levels in Female Mice. Front Endocrinol. 2019;10:758.

3. Uenoyama Y, Inoue N, Nakamura S, Tsukamura H. Central Mechanism Controlling Pubertal Onset in Mammals: A Triggering Role of Kisspeptin. Front Endocrinol (Lausanne). 2019;10:312.

4. Kotani M, Detheux M, Vandenbogaerde A, Communi D, Vanderwinden JM, Le Poul E, Brezillon S, Tyldesley R, Suarez-Huerta N, Vandeput F, et al. The metastasis suppressor gene KiSS-1 encodes kisspeptins, the natural ligands of the orphan G protein-coupled receptor GPR54. J Biol Chem. 2001;276(37):34631-6.

5. Kroll H, Bolsover S, Hsu J, Kim SH, Bouloux PM. Kisspeptin-evoked calcium signals in isolated primary rat gonadotropin- releasing hormone neurones. Neuroendocrinology. 2011;93(2):114-20.

6. Zhu HJ, Li SJ, Pan H, Li N, Zhang DX, Wang LJ, Yang HB, Wu Q, Gong FY: The Changes of Serum Leptin and Kisspeptin Levels in Chinese Children and Adolescents in Different Pubertal Stages. 2016, 2016:6790794.

7. Takumi K, Shimada K, lijima N, Ozawa H. Maternal high-fat diet during lactation increases Kiss1 mRNA expression in the arcuate nucleus at weaning and advances puberty onset in female rats. Neuroscience research. 2015;100:21-8.

8. Luo Q, Li W, Li M, Zhang X, Zhang H. Leptin/leptinR-kisspeptin/kiss1r-GnRH pathway reacting to regulate puberty onset during negative energy balance. Life Sci. 2016;153:207-12.

9. Majarune S, Nima P, Sugimoto A, Nagae M, Inoue N, Tsukamura H, Uenoyama Y. Ad libitum feeding triggers puberty onset associated with increases in arcuate Kiss1 and Pdyn expression in growthretarded rats. J Reprod Dev. 2019;65(5):397-406.

10. Nittari G, Scuri S, Petrelli F, Pirillo I, di Luca NM, Grappasonni I. Fighting obesity in children from European World Health Organization member states. Epidemiological data, medical-social aspects, and prevention programs. Clin Ter. 2019;170(3):e223-30.

11. Heidarianpour A, Shokri E, Baghian T, Shokri B. Benefits of aerobic training in girls with precocious puberty: involvement of CRP and cortisol. J Pediatr Endocrinol Metab. 2019;32(9):1005-11.

12. Amaro-Gahete FJ, Sanchez-Delgado G, Jurado-Fasoli L. Assessment of maximal fat oxidation during exercise: A systematic review. 2019, 29(7):910-921.

13. Paxinos G, Watson C: The Rat Brain Stereotaxic Co-Ordinates, vol. Massachusetts; 2007.

14. Feng J, Xu R, Li Y, Zhou Q, Song G, Deng Y, Yan Y. The effect of high-fat diet and exercise on KISS1/GPR54 expression in testis of growing rats. Nutr Metab (Lond). 2021;18(1):1.

15. Takumi K, lijima N, Ozawa H. Developmental changes in the expression of kisspeptin mRNA in rat hypothalamus. Journal of molecular neuroscience: MN. 2011;43(2):138-45. 
16. Rønnekleiv OK, Qiu J, Kelly MJ. Arcuate Kisspeptin Neurons Coordinate Reproductive Activities with Metabolism. Semin Reprod Med. 2019;37(3):131-40.

17. Reinehr T, Bosse C, Lass N, Rothermel J, Knop C, Roth CL. Effect of Weight Loss on Puberty Onset in Overweight Children. J Pediatr. 2017;184:143-50.e141.

18. Sitticharoon C, Mutirangura $P$, Chinachoti T, lamaroon A, Triyasunant N, Churintaraphan $M$, Keadkraichaiwat I, Maikaew P, Sririwichitchai R. Associations of serum kisspeptin levels with metabolic and reproductive parameters in men. Peptides. 2020;135:170433.

19. Matsuzaki T, Iwasa T, Kinouchi R, Yoshida S, Murakami M, Gereltsetseg G, Yamamoto S, Kuwahara A, Yasui T, Irahara M. Fasting reduces the kiss1 mRNA levels in the caudal hypothalamus of gonadally intact adult female rats. Endocr J. 2011;58(11):1003-12.

20. Rizzoto G, Sekhar D, Thundathil JC, Chelikani PK, Kastelic JP. Calorie Restriction Modulates Reproductive Development and Energy Balance in Pre-Pubertal Male Rats. Nutrients 2019, 11(9).

21. Howard SR, Guasti L, Poliandri A, David A, Cabrera CP, Barnes MR, Wehkalampi K, O'Rahilly S, Aiken $\mathrm{CE}$, Coll AP, et al. Contributions of Function-Altering Variants in Genes Implicated in Pubertal Timing and Body Mass for Self-Limited Delayed Puberty. J Clin Endocrinol Metab. 2018;103(2):649-59.

22. Busch AS, Højgaard B, Hagen CP, Teilmann G. Obesity Is Associated with Earlier Pubertal Onset in Boys. J Clin Endocrinol Metab 2020, 105(4).

23. Paltoglou G, Avloniti A, Chatzinikolaou A, Stefanaki C, Papagianni M, Papassotiriou I, Fatouros IG, Chrousos GP, Kanaka-Gantenbein C, Mastorakos G. In early pubertal boys, testosterone and LH are associated with improved anti-oxidation during an aerobic exercise bout. Endocrine. 2019;66(2):370-80.

\section{Figures}




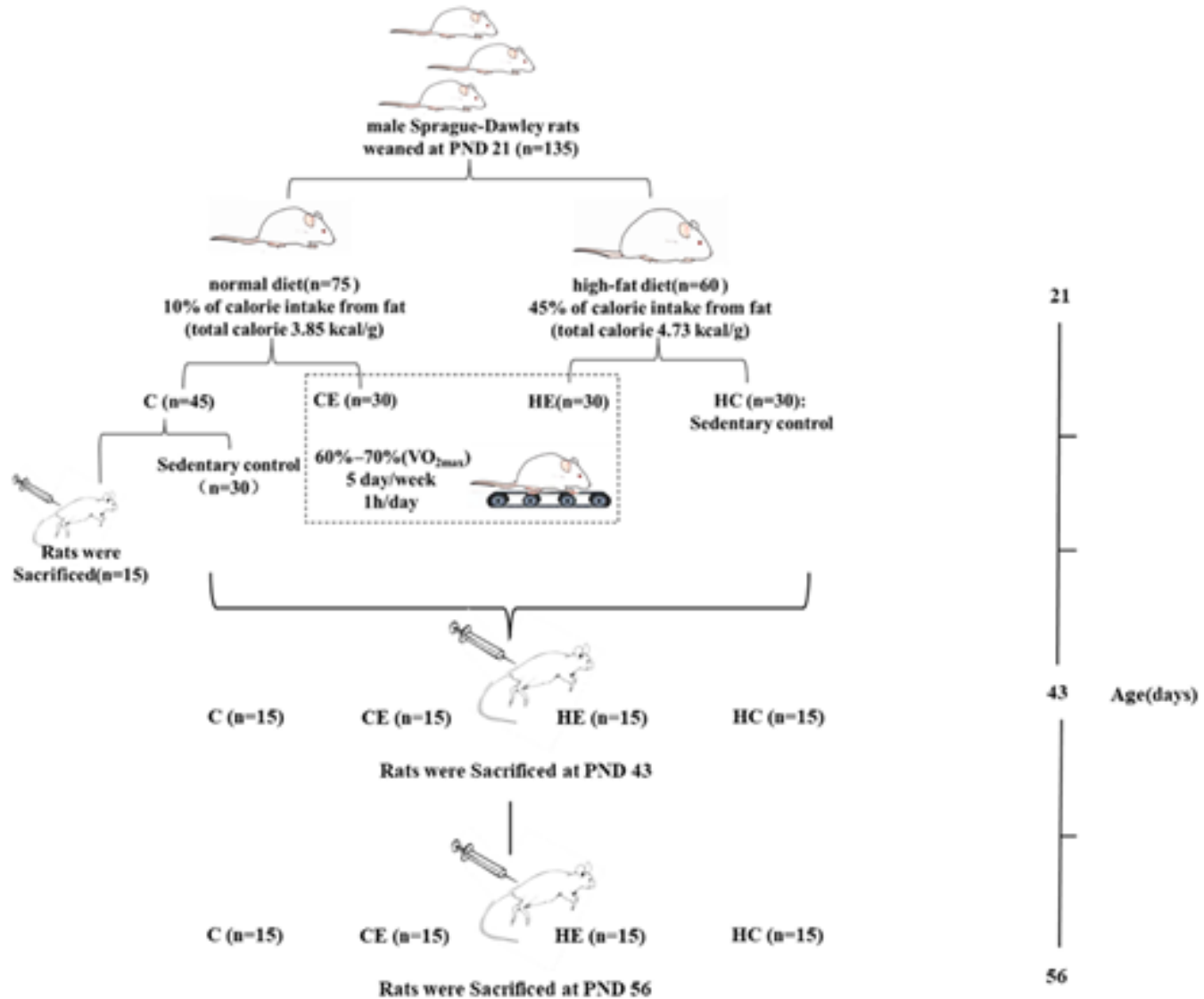

Figure 1

Experimental flow. 
A

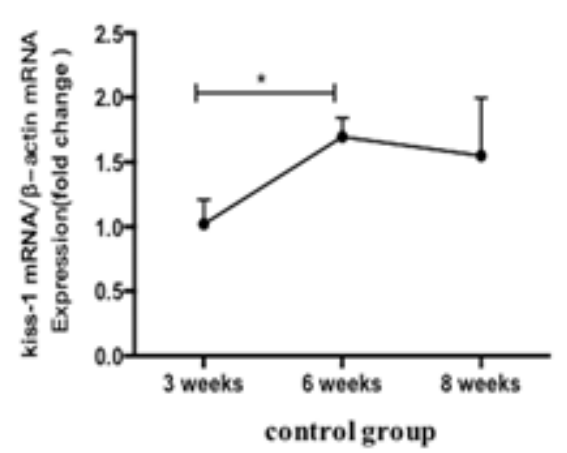

$\mathrm{C}$

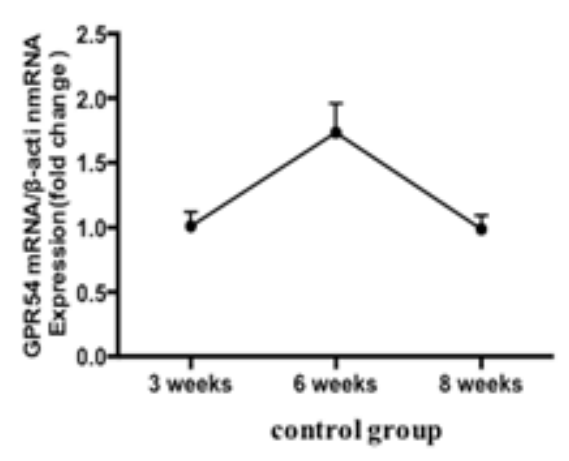

E
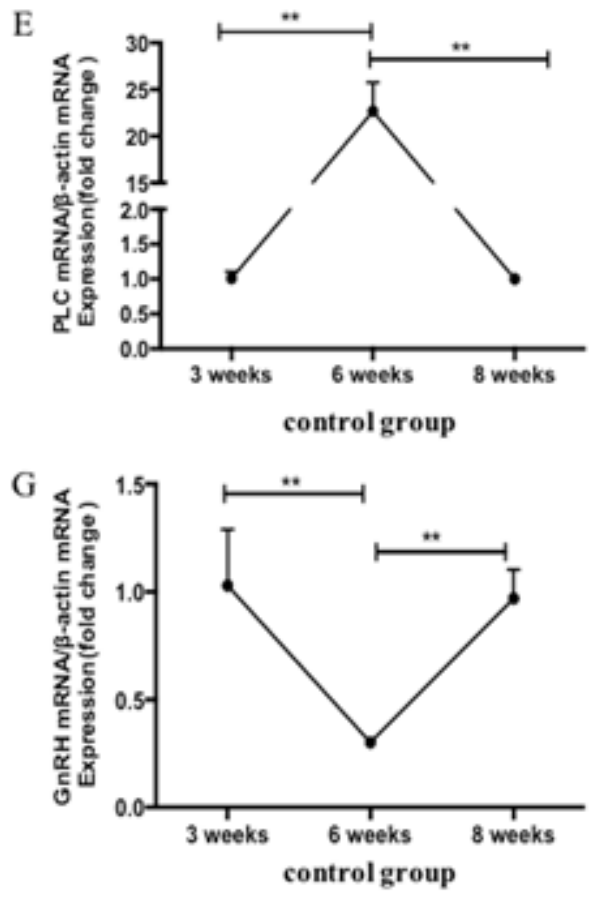

B

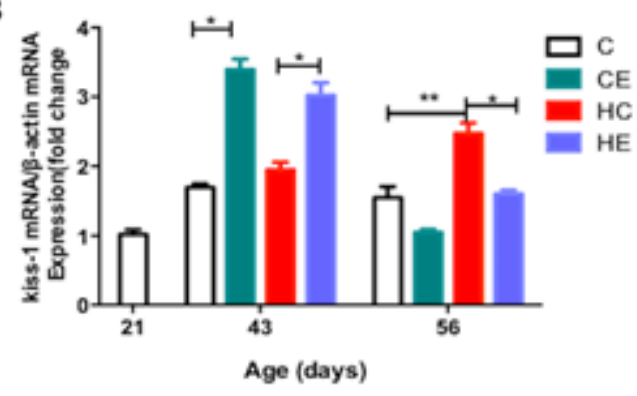

D

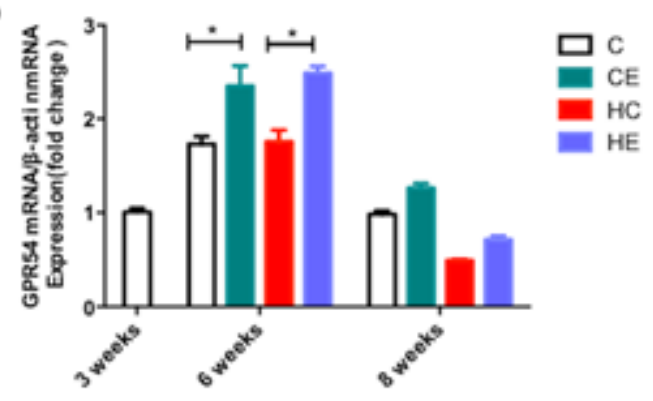

$\mathrm{F}$

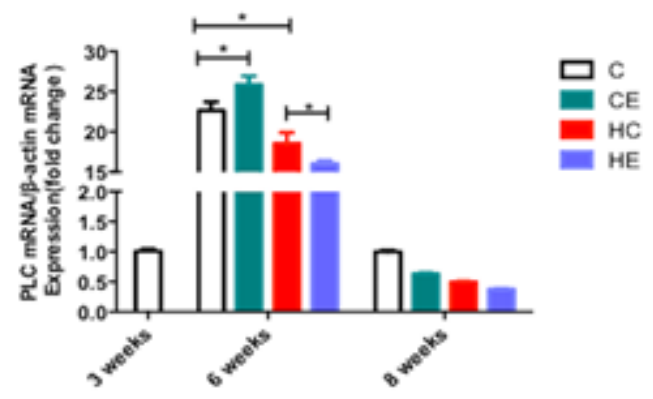

$\mathrm{H}$

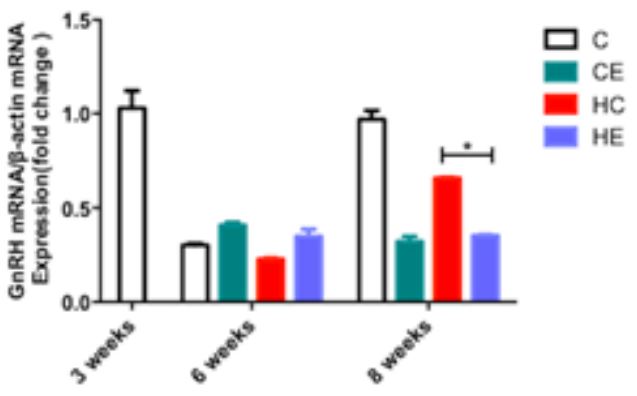

Figure 2

RT-qPCR analysis of Kiss-1, GPR54, PLC, and GnRH in the hypothalamic following a high-fat diet and $60 \%-70 \% \mathrm{~V}(\cdot) 02$ max treadmill training at PND 21st, 43rd, 56th. (A) Kiss-1 in the control group at PND $21 \mathrm{st}$, 43rd, 56th. (B) Effects of a high-fat diet and $60 \%-70 \% \mathrm{~V}(\cdot) 02 \mathrm{max}$ treadmill training on the expression of Kiss-1 mRNA in rats at PND 43rd, 56th. (C) GPR54 in the control group at PND 21st, 43rd, 56th. (D) Effects of a high-fat diet and 60\%-70\% V(•)02max treadmill training on the expression of GPR54 mRNA in rats at PND 43rd, 56th. (E) PLC in the control group at PND 21st, 43rd, 56th. (F) Effects of a hiah-fat diet and $60 \%-70 \% \mathrm{~V}(\cdot) 02$ max treadmill training on the expression of PLC mRNA in rats at Loading [MathJax]/jax/output/CommonHTML/fonts/TeX/fontdata.js 
PND 43rd, 56th. ( $n=6$ per group; error bar, mean \pm SEM.*, $P<0.05$ ). Statistical analyses were performed by assessing three-factor variances.

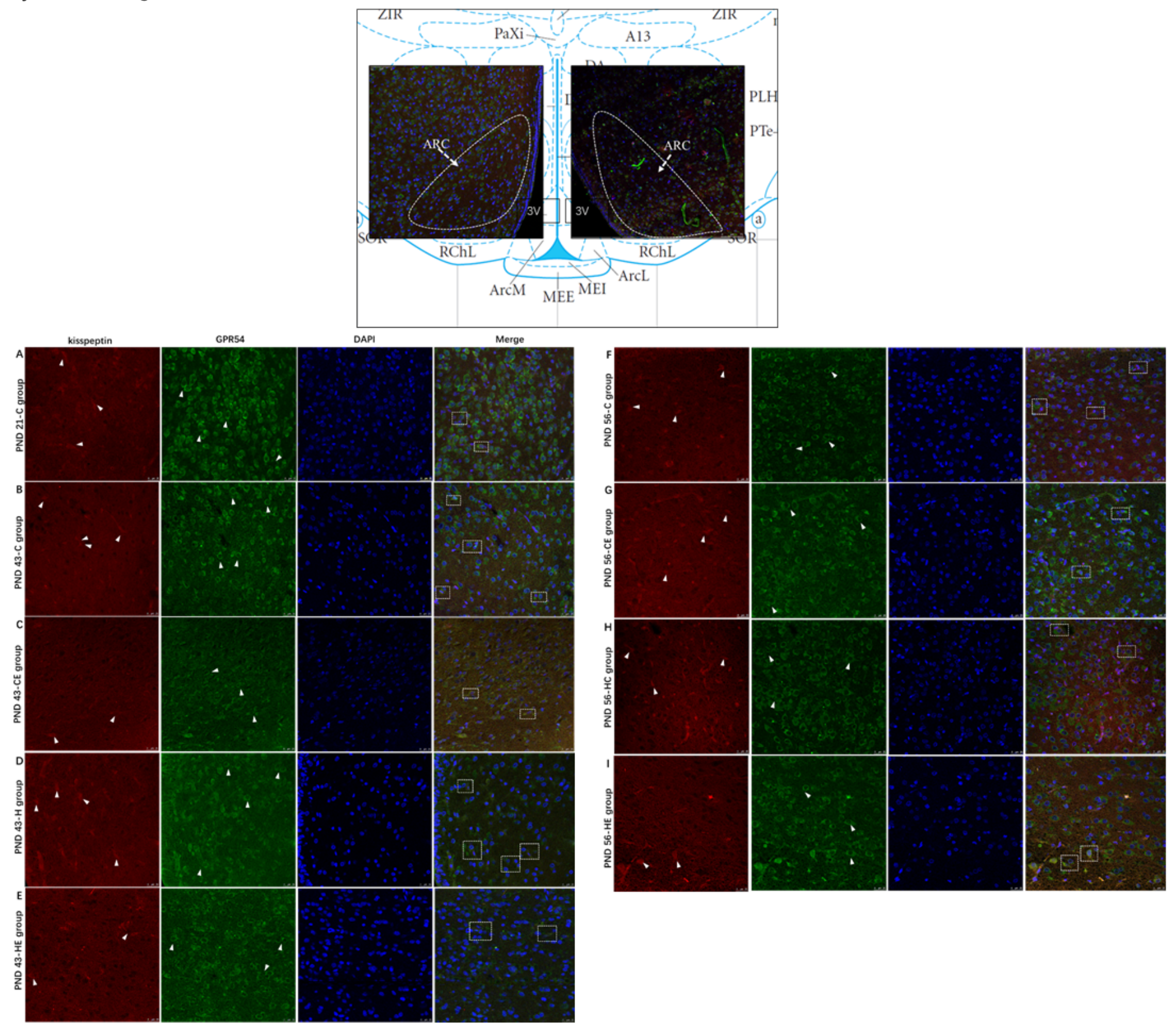

\section{Figure 3}

The majority of kisspeptin and GPR54 neurons are located in ARC within hypothalamic.

Immunofluorescent staining of Kiss-1 protein and GPR54 protein in the ARC of hypothalamic following a high-fat diet and $60 \%-70 \% \mathrm{~V}(\cdot) 02$ max treadmill training at PND 21st, 43rd, 56th(A-I). $\mathrm{n}=3$ per group, scale bar=25 $\mu \mathrm{m}$. 
A

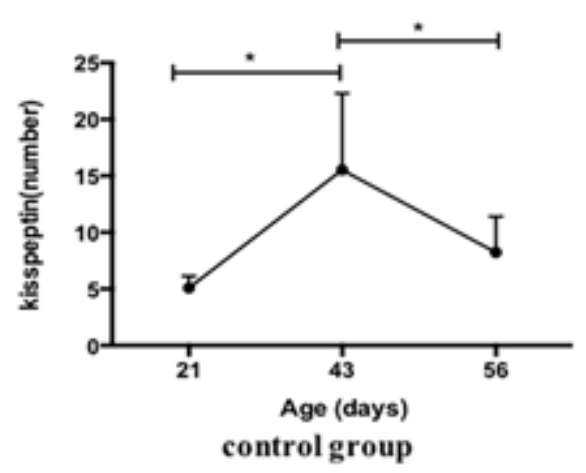

C

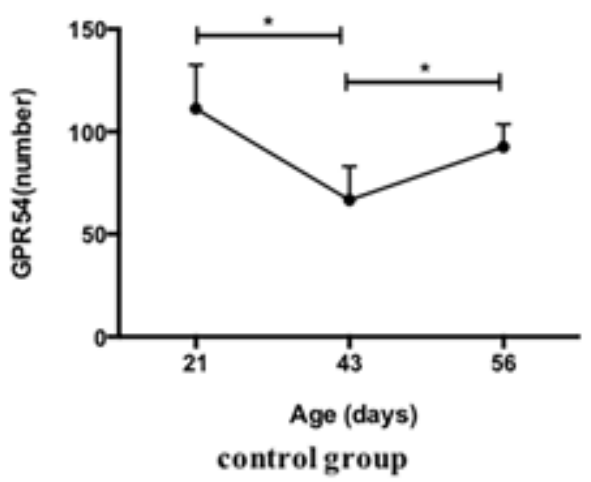

B

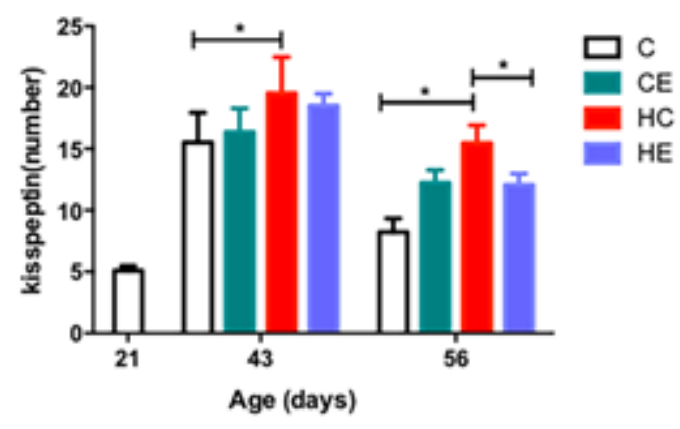

D

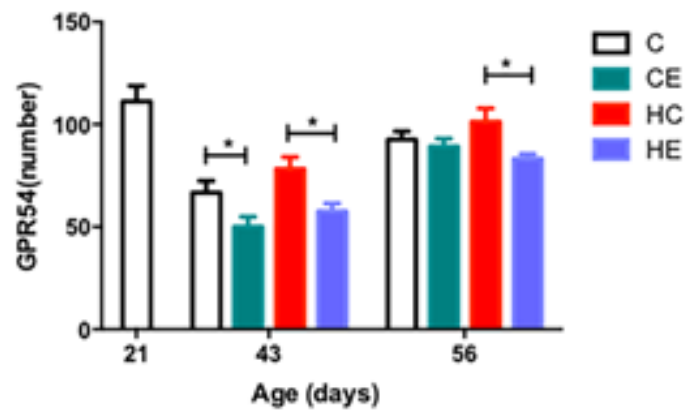

\section{Figure 4}

Immunofluorescent analysis in the hypothalamic ARC following a high-fat diet and $60 \%-70 \% \mathrm{~V}(\cdot) \mathrm{O} 2 \mathrm{max}$ treadmill training at PND 21st, 43rd, 56th. (A) Kiss-1 antibody in the hypothalamic ARC in the control group at PND 21st, 43rd, 56th. (B) Effects of a high-fat diet and $60 \%-70 \% \mathrm{~V}(\cdot) 02$ max treadmill training on the protein expression of Kiss-1 in rats at PND 43rd, 56th. (C) GPR54 antibody in the hypothalamic ARC in the control group at PND 21st, 43rd, 56th. (D) Effects of a high-fat diet and $60 \%-70 \% \mathrm{~V}(\cdot) 02 \mathrm{max}$ treadmill training on the protein expression of GPR54 in rats at PND 43rd, 56th. Statistical analyses were performed by assessing three-factor variances. ( $n=3$ per group; error bar, mean $\pm S E M . *, P<0.05$ ). 

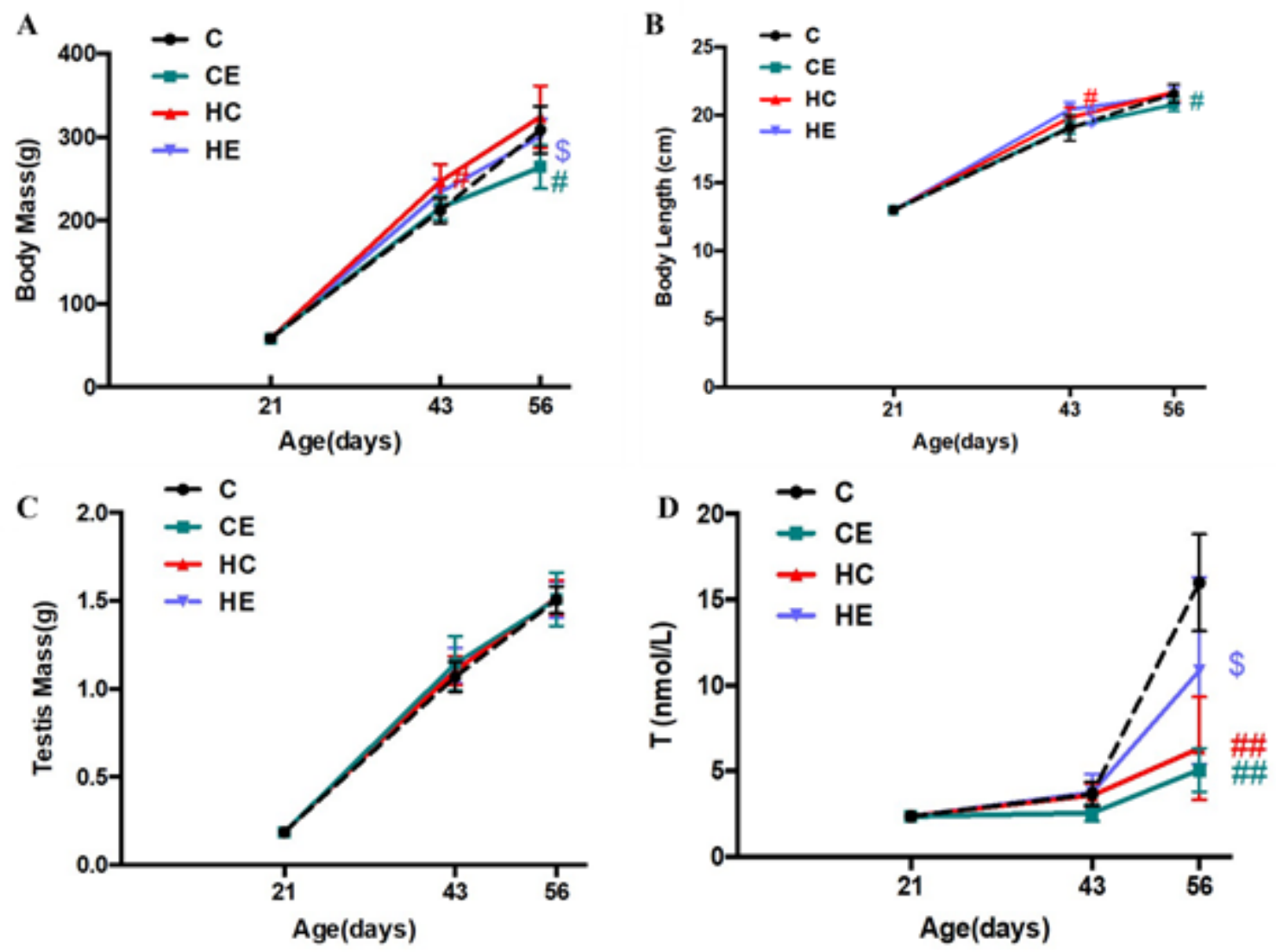

Figure 5

Effects of a high-fat diet and $60 \%-70 \% \mathrm{~V}(\cdot) 02$ max treadmill training on body mass and reproductive hormones in rats at PND 21st, 43rd, 56th. (A) Body mass changes at PND 21st, 43rd, 56th. (B) Bodylength changes at PND 21st, 43rd, 56th. (C) Testis weight changes at PND 21st, 43rd, 56th. (D) CLIAs for serum testosterone in rats at PND 21 st, 43 rd, 56th. \# P< 0.05 vs. C, \& $P<0.05$ vs. CE, $\$ P<0.05$ vs. HC.

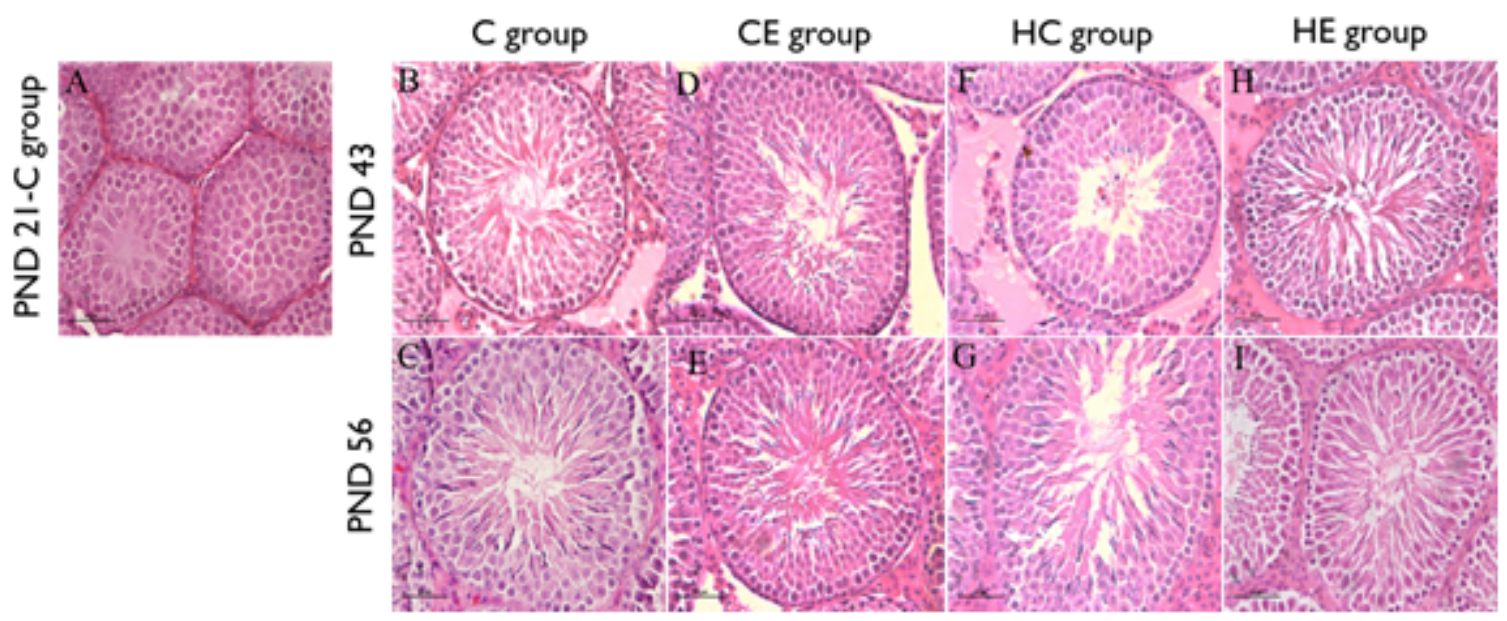

Figure 6

Effects of a high-fat diet and $60 \%-70 \% \mathrm{~V}(\cdot) 02$ max treadmill training on a testicular morphology in rats at PNID 21ct 12rd $5 \mathrm{kth}(\Delta-1) \mathrm{HF}$ ctainino of toctic in tho different groups. Statistical analyses were Loading [MathJax]/jax/output/CommonHTML/fonts/TeX/fontdata.js 
performed by assessing three-factor variances. $n=6$ per group, scale bar:50 $\mu \mathrm{m}$. 